\title{
LA AGRESIVIDAD AMBIENTAL Y LA DURABILIDAD DE LAS ESTRUCTURAS DE HORMIGON
}

(ENVIRONMENTAL AGGRESSIVENESS AND THE DURABILITY OF CONCRETE STRUCTURES)

\author{
B. Perepérez Ventura, Catedrático de Construcciones Arquitectónicas \\ E. Barberá Ortega, Profesor Titular de Construcciones Arquitectónicas \\ Universidad Politécnica de Valencia. \\ C. Andrade Perdrix, Profesor de Investigación \\ IETcC/CSIC
}

\section{RESUMEN}

El deterioro de las estructuras de hormigón es el resultado de la interacción entre ellas y el medio ambiente. En el presente trabajo se comentan los factores ambientales más determinantes y sus interacciones, con especial referencia a la corrosión de las armaduras, asi como diferentes propuestas de clasificación de la agresividad.

\section{SUMMARY}

The deterioration of concrete structures is the result of the interaction between them and the environment. In this study, comments are made on the most decisive environmental factors and their interactions. Special reference is made to the corrosion of the reinforcements and also to the different proposals of classification of the aggressiveness.

\section{INTRODUCCION}

Las estructuras se diseñan, calculan y construyen para que satisfagan unos requisitos mínimos de seguridad, funcionalidad y aspecto externo durante un período de tiempo, llamado "vida útil prevista", sin costes inesperados de mantenimiento o reparación (1). La capacidad de respuesta de la estructura depende de ciertos parámetros intrínsecos como las dimensiones de los elementos, los detalles constructivos y la calidad de los materiales, y de otros extrínsecos que, en definitiva, son función de las condiciones de servicio (2). Este segundo grupo es el sujeto a mayores dispersiones, en particular las condiciones ambientales (1), hecho transcendente en los problemas de durabilidad si se tiene presente que la causa principal de los deterioros estructurales hay que buscarla en la no consideración de la interacción estructura-medio ambiente, o al menos, en un análisis de la misma menos riguroso que el aplicado a la interacción estructura-cargas (3).
La intensidad y naturaleza de la agresividad ambiental viene determinada por los factores de contaminación y los metereológicos, que a menudo actúan simultáneamente, potenciándose sus efectos (4).

La definición de la agresividad ambiental en base a la simple ubicación geográfica de las construcciones entraña considerables riesgos de valoración, como han puesto de manifiesto diversas investigaciones $(5,6,7$, 8,9 ), ya que en ambientes de cierta agresividad, por ejemplo, la orientación de los elementos influye sobre el quebranto de la vida útil prevista $(10,11,12)$ y la dirección y velocidad del viento dominante es un factor importante al repercutir en la cantidad de humedad y contaminantes aportada localmente $(4,10,11,12)$.

La influencia respecto de la durabilidad del clima atmosférico general o macro-clima es menor que la del clima local (distancia en metros) o la del microclima (distancia en centímetros o milimetros) (2). En efecto, 
la humedad relativa cerca del suelo suele ser de un $10 \%$ a un $20 \%$ más elevada que en las proximidades del techo; en determinados locales (baños, cocinas, etc.) la humedad suele ser superior a las de las dependencias contiguas; hay elementos, como las placas de cubierta, que corren el riesgo de calentamiento diurno y de condensaciones superficiales nocturnas, lo que genera ciclos alternados de humidificación-desecación y, por tanto, la lixiviación de los álcalis y una carbonatación precoz del hormigón, que puede acortar sensiblemente la vida útil de tales elementos (13). En las partes de la estructura enterrradas o sumergidas, el mayor o menor flujo de oxígeno y humedad puede contribuir al desarrollo de procesos corrosivos en los que actúan de cátodo las zonas más ventiladas (13).

\section{FACTORES DETERMINANTES}

El acero embebido en un hormigón de buena calidad permanece pasivo durante prolongados períodos de servicio. Sin embargo, esta pasividad puede destruirse debido al ingreso de sustancias agresivas en el interior del hormigón.

Las sustancias agresivas que más frecuentemente producen la corrosión de las armaduras son los cloruros y el anhídrido carbónico del aire.

En ambientes marinos, los cloruros están presentes tanto en el propio agua de mar, como en la humedad de la atmósfera. Estos cloruros van penetrando a través de la red de poros y las fisuras del hormigón, y cuando alcanzan la armadura en cantidad suficiente, producen la rotura local de la capa pasivante del acero.

También los cloruros pueden producir daños muy serios por corrosión en ambientes no marinos, cuando se emplean como sales de deshielo. En este caso, se van acumulando en el interior del hormigón en cantidades crecientes cada invierno, reduciendo enormemente la vida útil de puentes y carreteras donde son usados. La vida media de un puente en las zonas frías de EE.UU. no sobrepasa los 15 años.

En cuanto al anhídrido carbónico de la atmósfera (y en general todas las sustancias ácidas), reacciona con las sustancias alcalinas del hormigón dando lugar a un descenso de su $\mathrm{pH}$ hasta valores que provocan la inmediata desaparición de la capa pasivante y, por tanto, posibilitan la corrosión del acero. La velocidad de ingreso del anhídrido carbónico depende, entre otros factores, de la porosidad del hormigón, y el espesor del recubrimiento puede resultar un factor determinante de la vida útil de la estructura. El fenómeno descrito se conoce con el nombre genérico de "carbonata- ción" y un valor medio de su velocidad de progreso viene dado por la expresión:

$$
x=K \sqrt{t}
$$

donde $X$ es el espesor carbonatado en un tiempo $t y$ $K$ es una constante de valores muy diferentes en función de la calidad del hormigón y la humedad ambiental.

\section{FACTORES ACELERANTES}

Los cloruros y el anhídrido carbónico son factores ambientales que pueden hacer cambiar las condiciones de servicio del hormigón, ya que determinan la corrosión o pasividad de las armaduras. Existen, en cambio, otros factores ambientales que no tienen ninguna influencia en la vida útil cuando el acero permanece pasivo, pero que cuando la corrosión se ha iniciado por otras causas (cloruros o carbonatación), lo que determinan es la cinética del proceso, es decir, controlan la velocidad de corrosión, pudiendo ser responsables de velocidades muy lentas o muy rápidas. Los facto. res ambientales más importantes como "acelerantes" del proceso de corrosión son: la humedad, el acceso de oxígeno hasta la armadura y la temperatura.

\section{Humedad}

La presencia de agua es imprescindible para la corrosión en medios neutros y alcalinos, pues interviene en el proceso catódico de reducción del oxígeno:

$$
2 \mathrm{H}_{2} \mathrm{O}+\mathrm{O}_{2}+4 \mathrm{e}^{-} 4 \mathrm{OH}^{-}
$$

Además, el agua es necesaria para la movilidad de los iones a través del electrólito. En el hormigón seco, la resistividad eléctrica es tan elevada que impide que la corrosión se produzca aun en ausencia de la capa pasivante sobre el acero; sólo la existencia de una cierta cantidad mínima de humedad en los poros del hormigón permitirá el desarrollo de los procesos corrosivos. Por tanto, cuanto más saturados en humedad estén los poros del hormigón, menor será el valor de la resistividad eléctrica y más elevadas podrán ser en principio las velocidades de corrosión.

El contenido en agua de los poros es función, en situaciones de no inmersión o no saturación, de la humedad relativa (HR) del ambiente. La humedad que importa, es decir, la que influye en los procesos de corrosión, es la realmente contenida en los poros. A este respecto, debe tenerse presente que la humedad ambiental y la del hormigón sólo coinciden en regímenes estacionarios y que en condiciones cambiantes es mayor la humedad del hormigón, porque éste pierde agua 


\begin{tabular}{|c|c|c|c|c|c|}
\hline \multirow{2}{*}{$\begin{array}{l}\text { Humedad relativa } \\
\text { efectiva. }\end{array}$} & \multicolumn{5}{|c|}{ Proceso } \\
\hline & $\begin{array}{l}\text { Carbona- } \\
\text { tacion }\end{array}$ & $\begin{array}{l}\text { Corros } \\
\text { las ar } \\
\text { ras er } \\
\text { hormi } \\
\text { causa } \\
\text { Carbo } \\
\text { nata - } \\
\text { cion. }\end{array}$ & $\begin{array}{l}\text { ión de } \\
\text { madu- } \\
\text { iel } \\
\text { gón } \\
\text { da por: } \\
\text { Conta- } \\
\text { mina- } \\
\text { ción } \\
\text { por clo } \\
\text { ruros }\end{array}$ & $\begin{array}{c}\text { Atoque } \\
\text { por } \\
\text { heladas }\end{array}$ & $\begin{array}{l}\text { Ataque } \\
\text { químico }\end{array}$ \\
\hline Muy baja $(<45 \%)$ & 1 & 0 & 0 & 0 & 0 \\
\hline Baja $(45-65 \%)$ & 3 & 1 & 1 & 0 & 0 \\
\hline Media $(65-85 \%)$ & 2 & 3 & 3 & 0 & 0 \\
\hline Alta $(85-95 \%)$ & 1 & 2 & 3 & 2 & 1 \\
\hline Saturación(>95\%) & 0 & 1 & 1 & 3 & 3 \\
\hline
\end{tabular}

$0=$ no significativo. $1=$ Bajo. 2 = Medio. 3 = Alto.

Fig. 1.-Influencia del contenido de humedad del hormigón sobre algunos procesos de deterioro.

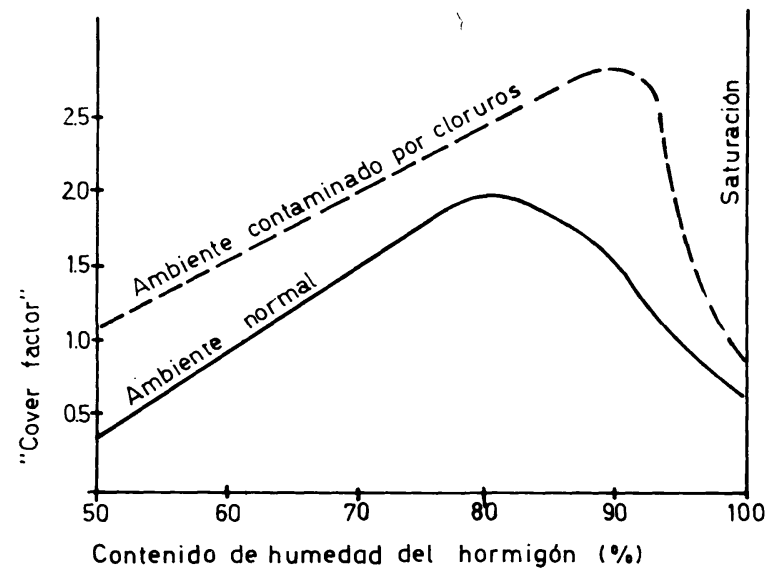

Fig. 2.-Influencia de la humedad relativa efectiva sobre el riesgo de corrosión de las armaduras.

a menor velocidad de lo que la gana a causa de la existencia de tensiones capilares. Este último fenómeno puede verse acrecentado cuando el hormigón esté contaminado por cloruros, a causa del carácter higrocópico de los mismos. Asimismo, aunque el ambiente exterior esté seco, el interior del hormigón no se seca más que cuando tales circunstancias se prolongan largo tiempo. En la mayoría de los hormigones, a partir de

los $3-4 \mathrm{~cm}$ del parámetro exterior, los poros siempre se encuentran saturados o casi saturados de humedad. Este comportamiento está muy influenciado por la porosidad del hormigón, ya que hormigones muy porosos permitirán una "respiración" más profunda que hormigones más densos, cuya "piel" sólo "respirará" en los $1-2 \mathrm{~cm}$ más externos.

A título de ejemplo se muestran en las figuras 1 (2) y 2 (2) distintas interpretaciones de la influencia que la humedad contenida en los poros puede tener en los procesos de corrosión de las armaduras. La figura 2 muestra en abscisas la humedad realmente contenida en los poros (nivel $0 \%$ de la saturación de los mismos), y en ordenadas se ha definido la escala de agresividad de tal manera que resulte directamente proporcional al espesor del recubrimiento necesario para mantener constante el riesgo de corrosión del acero.

\section{Acceso de oxigeno}

Tampoco es posible que se desarrolle el proceso de corrosión sin que llegue una mínima cantidad de oxigeno hasta las armaduras, es decir, sin una cierta "aireación" de las mismas.

Durante mucho tiempo, e incluso con cierta frecuencia en la actualidad, se ha considerado que el acceso o flujo de oxígeno es el factor determinante de la velocidad de corrosión. Así, se piensa a menudo que el espesor del recubrimiento influye mucho en el acceso de oxígeno, y se han realizado múltiples trabajos para medir la permeabilidad del hormigón al oxígeno, el aire 0 los gases. Pero tal creencia es errónea y tampoco son válidas las extrapolaciones al fenómeno de la corrosión de las armaduras de los ensayos de permeabilidad del hormigón, cuando se utiliza la presión mecánica como fuerza impulsora de los gases.

Si el flujo de oxígeno fuera el factor determinante de la velocidad de corrosión, sin duda ésta debería ser mayor en un hormigón seco (caso 1, figura 3), conservado por ejemplo en una atmósfera con un $50 \%$ de $\mathrm{HR}$, que un hormigón húmedo donde el flujo de oxígeno es menor ya que éste tiene que disolverse previamente en el agua contenida en los mismos. Sin embargo, la evidencia experimental muestra claramente que la velocidad de corrosión es máxima cuando los poros tienen suficiente agua para facilitar los fenómenos de corrosión y, por otro lado, no están saturados (caso 2, figura 3).

Cuando el hormigón tiene los poros completamente saturados de humedad (caso 3, figura 3), el oxígeno se tiene que disolver en el agua antes de alcanzar las cercanías de la armadura. En este caso sí que el flujo de oxígeno es el factor controlante, ya que la cantidad que fluye es muy limitada. En todos los demás casos, el acceso de oxígeno siempre es suficiente para soportar la velocidad de corrosión que permita la resistividad del hormigón.

Es necesario recordar aquí lo dicho para el factor humedad y la capacidad de "respiración" del hormigón; cuando el hormigón es denso y el ambiente exterior contiene unos niveles medios de humedad suficientes, los poros están completamente saturados de agua a partir de los $3.4 \mathrm{~cm}$ del exterior. Por tanto, si hay armaduras más interiores y éstas están despasivadas, su velocidad de corrosión estará limitada, pero si están más 


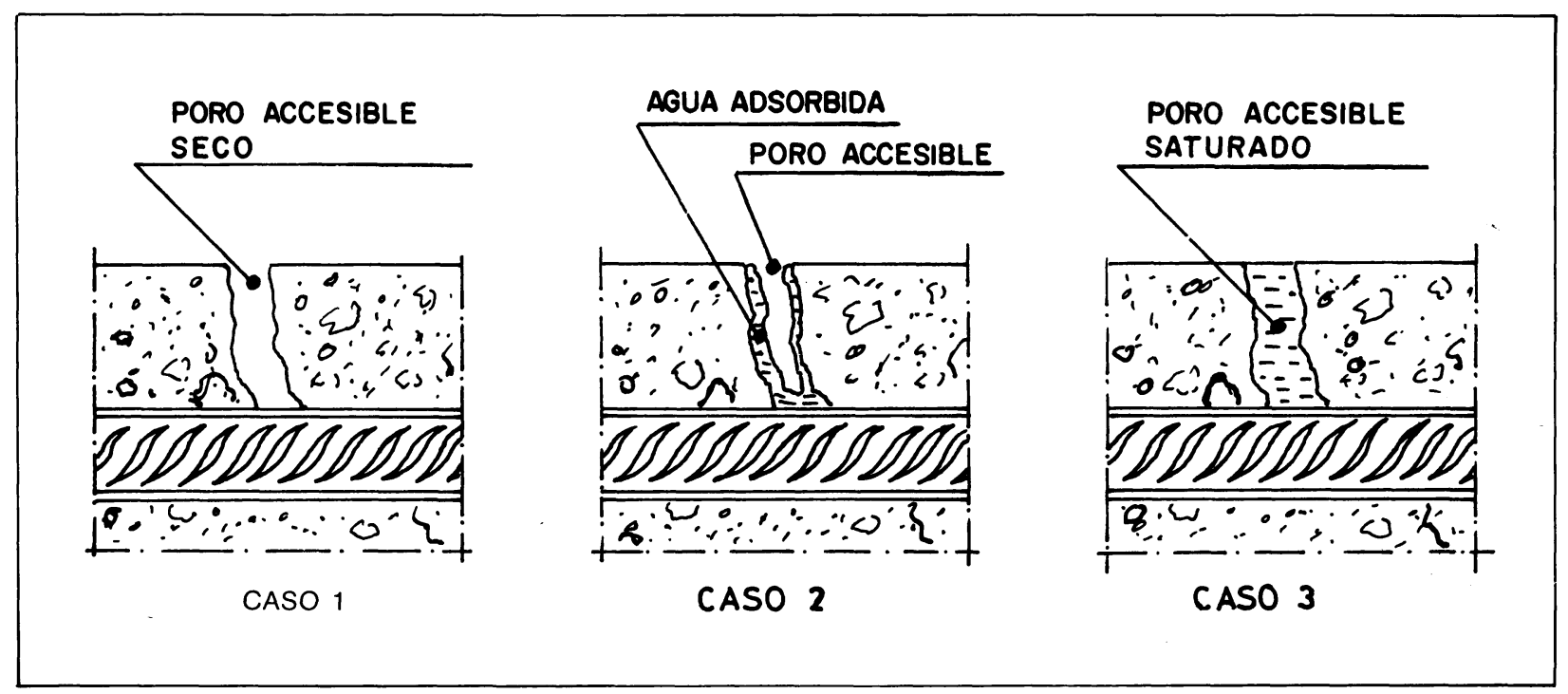

Fig. 3.-Poros accesibles del hormigón. Ejemplos de diversos contenidos de humedad.

cerca de la superficie, su velocidad de corrosión puede ser muy elevada.

En cuanto a la no validez de los ensayos en los que se intenta correlacionar la mayor o menor permeabilidad del hormigón a los gases (utilizando presiones mecánicas como fuerzas impulsoras) con su menor o mayor capacidad protectora en procesos de corrosión, han sido Gjörv y Vennesland (14) los pioneros en poner de manifiesto que, como la corrosión es un proceso electroquímico, sólo son válidos los valores de flujo de oxigeno cuando se miden utilizando "fuerzas impulsoras" de tipo electroquímico. Así, estos autores han propuesto un tipo de ensayo en el que miden el flujo de oxígeno mediante las intensidades de reducción catódica del mismo (a $-750 \mathrm{mv}$ respecto del electrodo de calomelanos saturado) sobre armaduras de acero. En estas circunstancias, han comprobado que los flujos de oxígeno medidos no coinciden con los deducidos con los otros tipos de ensayos.

Una aproximación grosera, pero suficiente y no sofisticada, para conocer la mayor o menor bondad de un hormigón en relación a su permeabilidad al oxígeno, es medir su porosidad o índice de huecos accesibles.

\section{Temperatura}

La temperatura juega también un papel doble en los procesos de deterioro. Por un lado, su incremento promociona la movilidad de las moléculas facilitando el transporte de sustancias (15); por otro, su disminución puede dar lugar a condensaciones que, a su vez, pueden producir incrementos locales importantes del contenido de humedad del material (13). Además, la cantidad absoluta de vapor de agua en la atmósfera varía con la temperatura.
La figura 4 (2), en la que en ordenadas se ha definido el riesgo de corrosión de las armaduras de igual modo que en la figura 2, permite deducir que, al pasar de una temperatura media anual de $5^{\circ} \mathrm{C}$ a otra de $20^{\circ} \mathrm{C}$, hay que doblar aproximadamente el espesor del recubrimiento de hormigón para mantener constante el riesgo de corrosión de las barras. El análisis de estructuras deterioradas por corrosión de las armaduras (3) ha corroborado el fenómeno, demostrando que, a igualdad de todos los demás condicionantes, los climas son tanto más agresivos cuanto más cálidos, y que entre los $20^{\circ} \mathrm{C}$ y los $40^{\circ} \mathrm{C}$ se producen aumentos bruscos de la velocidad de corrosión, en particular en presencia de humedades relativas relativamente elevadas.

Finalmente, es importante destacar que estos tres factores ambientales tienen efectos contrapuestos y que, por tanto, no es fácil predecir la evolución del proceso de corrosión del acero a partir de uno solo de ellos. Esta característica del "efecto inversor" (por ejemplo, una mayor humedad facilita la corrosión pero impide el ac-

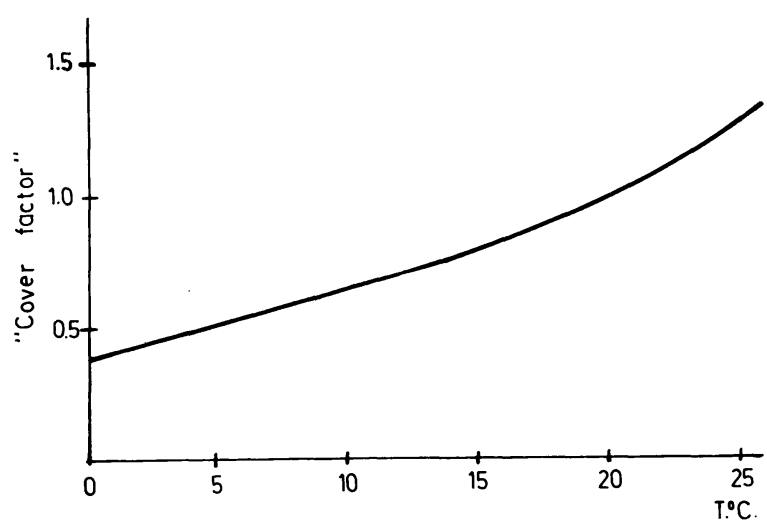

Fig. 4.-Influencia de la temperatura sobre el riesgo de corrosión de las armaduras. 
ceso de oxígeno, o bien una mayor temperatura acelera la corrosión pero disminuye la condensación), lleva a múltiples predicciones erróneas de comportamiento de las armaduras.

\section{CLASIFICACION DE LA AGRESIVIDAD AMBIENTAL}

A partir de los conocimientos previamente expuestos, se ha intentado definir en distintas normativas los niveles de agresividad ambiental. Esta clasificación no resulta nada fácil, ya que una clasificación prolija y completa que tenga en cuenta todos los posibles efectos ya mencionados y la acción de los microclimas, puede resultar inviable de aplicar y, por el contrario, un intento simplificador tiene el riesgo de no contemplar situaciones particulares.

A título de ejemplo se dan a continuación algunas clasificaciones ambientales contenidas en la normativa española y en el Código Modelo del CEB. Particularmente sucinta es la clasificación que ofrece la Instrucción EH-82 (16) (figura 5), con un planteamiento similar al que realiza el Model Code (17) (figura 6), si bien las definiciones de éste son algo más explícitas. También se muestra en la figura 7 la clasificación aprobada por el T.G. $n .^{\circ} 20$ del CEB (2) obtenida a partir de la propuesta formulada por el prEN 206 (figura 8) (2).

La intención de éstas u otras clasificaciones es agrupar ambientes característicos que pueden representar niveles de agresividad similares. A continuación se expone un intento de sistematización de la complejidad de ambientes con que pueden encontrarse en la práctica los profesionales de la construcción.

\begin{tabular}{|l|l|}
\hline CASO I & ELEMENTOS INTERIORES EN AMBIENTE NORMAL \\
\hline CASO II & $\begin{array}{l}\text { ELEMENTOS INTERIORES EN AMBIENTE HUMEDO O MEDIANAMENTEE } \\
\text { AGRESIVOS Y ELEMENTOS EXTERIORES A LA INTEMPERIE }\end{array}$ \\
\hline CASO III & $\begin{array}{l}\text { ELEMENTOS INTERIORES O EXTERIORES EN AMBIENTE MUY AGRE- } \\
\text { SIVO OUE OEBAN ASEGURAR CA ESTANQUEIOAD }\end{array}$ \\
\hline
\end{tabular}

Fig. 5.-Clasificación de la agresividad ambiental según la IEH-82.

\begin{tabular}{|c|c|}
\hline $\begin{array}{l}\text { AMBIEN TE } \\
\text { NO AGRESIVO }\end{array}$ & $\begin{array}{l}\text { INTERIORES DE LAS VIVIENDAS U OFICINAS } \\
\text { MEDIOS DONDE SOLO SE ALCANCE UN VALOR ELEVADO JE LA H.R. DU- } \\
\text { RANTE CORTOS PERIODOS DE TIEMPO (P.e...60\% de HR duranile mems de } \\
\text { tres meses al año). }\end{array}$ \\
\hline $\begin{array}{l}\text { AMBIENTE } \\
\text { MODERADAMENTE } \\
\text { AGRESIVO }\end{array}$ & $\begin{array}{l}\text { INTERIORES DE EDIFICIOS EN LOS QUE LA HUMEDAD ES ELEVADA O } \\
\text { EN DONDE SE TEME LA APARICION TEMPORAL DE VAPORES CORRO- } \\
\text { SIVCS. }\end{array}$ \\
\hline $\begin{array}{l}\text { AMBIENTE } \\
\text { AGRESIVO }\end{array}$ & 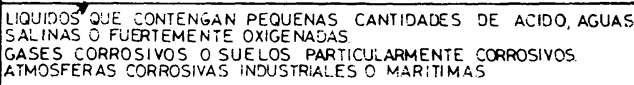 \\
\hline
\end{tabular}

Fig. 6.-Clasificación de la agresividad ambiental según el Model Code. (C) Consejo Superior de Investigaciones Científicas Licencia Creative Commons 3.0 España (by-nc)

\begin{tabular}{|c|c|c|}
\hline \multicolumn{2}{|c|}{$\begin{array}{l}\text { CLASE DE } \\
\text { EXPOSICION }\end{array}$} & CONDICIONES AMBIENTALES \\
\hline \multicolumn{2}{|l|}{1} & $\begin{array}{l}\text { AMBIENTE SEDO LOCALES GENERALMENTE SECOS CON HR. CASI CONS- } \\
\text { TANTE QUE SOL DE MODO INFRECUENTE SUPERA EL } 70 \% \text { (P.e. miterio- } \\
\text { res de viviendas U oficinas) }\end{array}$ \\
\hline \multirow{2}{*}{2} & a & \multirow{2}{*}{ 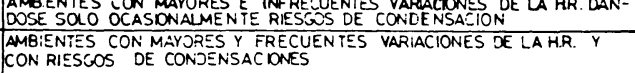 } \\
\hline & $b$ & \\
\hline \multicolumn{2}{|l|}{3} & 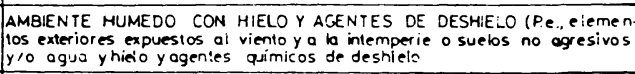 \\
\hline \multicolumn{2}{|c|}{4} & 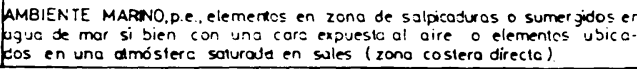 \\
\hline
\end{tabular}

Fig. 7.-Clasificación de la agresividad ambiental respecto de la durabilidad de las armaduras.

\begin{tabular}{|c|c|c|}
\hline \multicolumn{2}{|c|}{$\begin{array}{l}\text { CLASE DE } \\
\text { EXPOSICION }\end{array}$} & CONDICIONES AMBIENTALES \\
\hline \multicolumn{2}{|c|}{1} & $\begin{array}{l}\text { CONDICIONES SECAS, COMO PE } \\
\text { - INTERIORES DE EDIFICIOS DE VIVIENDAS Y OFICINAS } \\
\text { - ELEMENTOS EXTERIORES NO EXPUESTOS A VIENTO Y AGUAO } \\
\text { SUELOSO AGUA. } \\
\text { - UBICACIONES CON HR MAYORES TAN SOLO POR CORTOS } \\
\text { PERIODOS DE TIEMPO }(P \text { C }>60 \% \text { CE HR menOS de } 3 \text { meSES } \\
\text { al año. }\end{array}$ \\
\hline \multirow[t]{2}{*}{2} & a & $\begin{array}{l}\text { AMBIENTE HUMEDO SIN HELADAS ( } 1 \text { ), PE } \\
\text { - INIERIORES DE EDIFICIOS CON HUMEDADES ELEVADAS. } \\
\text { - ELEMENTOS EXTERIORES EXPUESTOS A VIENTO Y AGUA PERO NO } \\
\text { AHELADAS. } \\
\text { - ELEMENTOS EN SUELOS NO AGRESIVOS YIO AGUA (Sin helOCOS) }\end{array}$ \\
\hline & b & $\begin{array}{l}\text { AMBIENTES HUMEDOS CON HIELO, PE } \\
\text { - ELEMENTOS EXTERIORES EXPUESTOS A VIENTO Y AGUA O } \\
\text { SUELOS NO AGRESIVOS YIO AGUA, (CON helOdOS) }\end{array}$ \\
\hline \multicolumn{2}{|c|}{3} & $\begin{array}{l}\text { AMBIENTES HUMEDOS CON HELADAS Y AGENTES DE DESHIELO. } \\
\text { PE: } \\
\text { - ELEMENTOS EXTERIORES EXPUESTOS A VIENTO Y AGUAOO } \\
\text { SUELOS NO AGRESIVOS YIO AGUA, CON HELADAS Y AGENTES } \\
\text { DE DESHIELO. }\end{array}$ \\
\hline \multirow[t]{2}{*}{4} & a & $\begin{array}{l}\text { AMBIENTES MARINOS, P. } \\
\text { - ELEMENTOS EN ZONAS DE SA:PICADURAS O SUMERGIDOS EN } \\
\text { AGUA DE MAR CON UNA CARA EXPUESTA A AIRE. } \\
\text { - ELEMENTOS EN UN AIRE SATURADO DE SALES (área Costero } \\
\text { directo). }\end{array}$ \\
\hline & b & $\begin{array}{l}\text { AMBIENTES MARINOS CON HELAOAS, P E: } \\
\text { - IDEM CASO ANTERIOR }(4-a) \\
\text { - IDEM CASO ANTERIOR }(4-0)\end{array}$ \\
\hline \multicolumn{3}{|r|}{ ESTAS OTRAS CLASES, SOLAS O COMBINADAS CON LAS ANTERIORES } \\
\hline \multirow{3}{*}{$\begin{array}{c}5 \\
(2)\end{array}$} & a & AMBIENTE OUIMICO LIGERAMENTE AGRESIVO (gas.líquido o sólico) \\
\hline & b & AMBIENTE QUIMICO MODERADAMENTE AGRESIVO (gas. líquido c sólido) \\
\hline & c & AMBIENTE QUIMICO ALTAMENTE AGRESIVO (gCS. líquico o sólido) \\
\hline \multicolumn{2}{|c|}{$\begin{array}{l}\text { (1) } \\
\text { (2) }\end{array}$} & $\begin{array}{l}\text { BAJO CONDICIONES EUROPEAS MODERADAS } \\
\text { VER CLASIFICACION ISO DE LAS CONDICIONES AMBIENTALES } \\
\text { QUIMICAMENTE AGRESIVAS PARA EL HORMGGN }\end{array}$ \\
\hline
\end{tabular}

Fig. 8.-Clasificación de la agresividad ambiental según prEN 206 del CEN.

\section{Ambientes interiores}

Aunque se consideran así todos aquellos que están aislados de la intemperie, lo cierto es que pueden darse en ellos grados importantes de agresividad. Tal puede ser el caso de aparcamientos subterráneos (con niveles de anhídrido carbónico muy elevados) o el caso de cocinas y cuartos de baño sometidos a elevadas condensaciones y humedades. Por tanto, al referirse a "interiores" habría que considerar como tales sólo aquellos ambientes con ausencia de sustancias que puedan provocar la corrosión de las armaduras y humedades relativas habitualmente bajas.

\section{Intemperie}

La variabilidad de los ambientes exteriores suele ser mucho mayor aún que los de los interiores, en particular http://informesdelaconstruccion.revistas.csic.es 
en el aspecto micro-climático. Habría, pues, que considerar que se trata de ambientes donde puede haber elevadas HR pero donde no hay niveles de cloruros apreciables.

\section{Ambiente marino}

La durabilidad del hormigón en ambiente marino es de especial interés; por un lado, porque mares y océanos ocupan el $80 \%$ del globo y buena parte de las actividades humanas se han ubicado en zonas costeras, siendo ésta una dinámica creciente, y por otro porque el hormigón es el material más durable y económico en el ambiente que nos ocupa (18).

La agresividad del ambiente marino se debe en parte al incremento de humedad que puede generar y, en particular, a las sales que lleva disueltas el agua de mar, cuyas concentraciones iónicas medias correspondientes a las sales más frecuentes, se muestran en la figura 9 (10). De entre estas sales, destaca el ión cloruro, responsable del mayor número de casos que se conocen de corrosión de las armaduras (18).

\begin{tabular}{|l|c|c|}
\hline $\begin{array}{c}\text { Nombre del } \\
\text { ión }\end{array}$ & $\begin{array}{c}\text { Abreviatura } \\
\text { química }\end{array}$ & $\begin{array}{c}\text { Concentracion } \\
\% \text { en peso }\end{array}$ \\
\hline Cloruro & $\mathrm{Cl}^{\prime \prime}$ & 19.35 \\
Sodio & $\mathrm{Na}^{+}$ & 10.76 \\
Sufato & $\mathrm{So}_{\mathrm{*}}^{+}$ & 2.71 \\
Magnesio & $\mathrm{Mg}^{+*}$ & 1.29 \\
Calcio & $\mathrm{Ca}^{+*}$ & 0.41 \\
Potasio & $\mathrm{K}^{+}$ & 0.39 \\
Otros & & $\frac{0.23}{35.16}$ \\
TOTAL & & \\
\hline
\end{tabular}

Fig. 9.-Concentraciones iónicas habituales en el agua de mar.

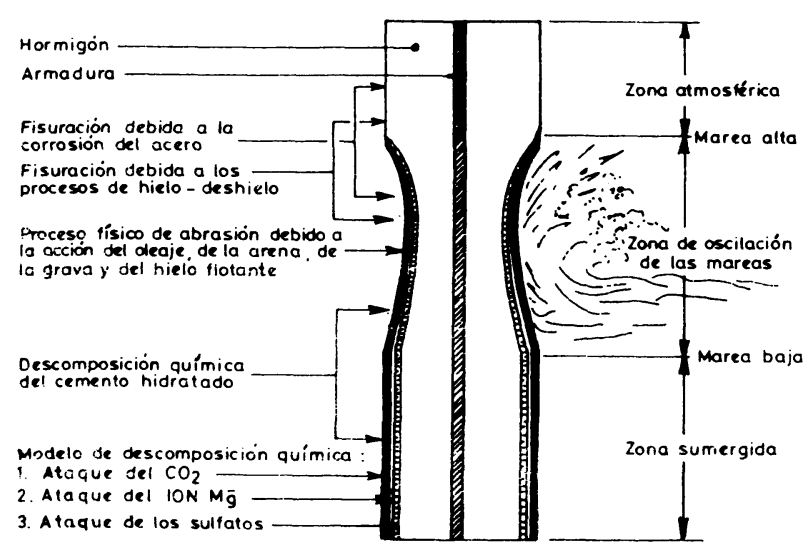

Fig. 10.-Procesos de deterioro del hormigón en ambiente marino.
La experiencia ha demostrado que los procesos individualizados de deterioro tienden a especializarse en las diferentes partes de la estructura, tal y como muestra la figura $10(18)$, pudiendo establecerse diferentes tipos de exposición (2):

a) Zona atmosférica, en la que la estructura recibe, aún a pesar de no estar en contacto con el agua, las sales. El nivel de cloruros depende de la distancia al mar y de la altura, sin olvidar la velocidad y dirección de los vientos y otros condicionantes geográficos.

b) Zona de salpicaduras, en la que se produce una acción directa del agua de mar, a causa del oleaje y de las salpicaduras que se derivan de su impacto sobre determinados obstáculos o las propias construcciones.

c) Zona de oscilación de las mareas, que es la limitada por los niveles máximo y mínimo alcanzados por las mareas y en la que el hormigón puede verse permanentemente saturado y con una acumulación creciente de sales.

d) Zonas sumergidas, o parte de la construcción situada por debajo del nivel de la marea baja y, por tanto, en régimen de inmersión permanente.

e) Zona de lecho marino, o parte de la estructura enterrada en el lecho marino.

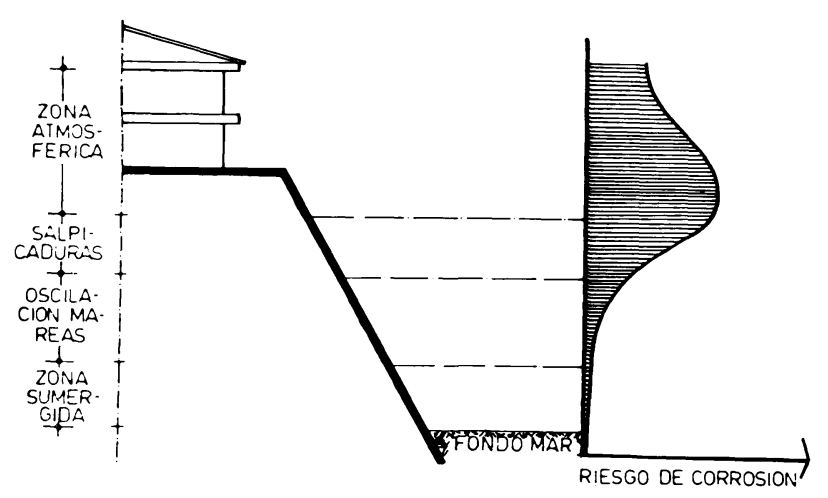

Fig. 11.-Tipos de exposición marina y variación del riesgo de corrosión de las armaduras. 
La variación del riesgo de corrosión de las armaduras se ha ilustrado en el conocido esquema de la figura 11, donde se observa que el riesgo es máximo en la zona atmosférica y en la de salpicaduras (10), tal y como también preconiza la figura 7 (2). En la zona atmosférica, la de mayor interés en el campo de la edificación, tal como indica la figura 11 , se produce una disminución del riesgo de corrosión con la altura del edificio respecto del nivel del mar, hecho contrastado en situaciones concretas (11). De hecho, el "ACl Committee 201" (19) permite pasar de una relación agua/cemento de 0,40 a otra de 0,50 cuando la edificación se sitúa al menos a ocho metros por encima del nivel del mar o en la zona de salpicaduras, o a más de treinta metros en horizontal.

\section{Sales de deshielo}

El uso de sales de deshielo da lugar a vidas en servicio de estructuras de hormigón aún más cortas que las de las situadas en ambientes marinos, ya que las cantidades de cloruros que se pueden acumular en el interior del hormigón llegan a ser muy elevadas. Por tanto, éste es uno de los ambientes más agresivos que pueden darse en relación al hormigón armado y pretensado. En nuestro país, donde el número de autopistas y carreteras en general ha crecido en los últimos tiempos, los casos de corrosión prematura de puentes en las zonas del norte empiezan a suponer una preocupación económica de primera magnitud.

\section{Hormigón en contacto con suelos}

Los suelos constituyen un medio extraordinariamente complejo. En relación a la posible corrosión de las armaduras lo que hay que tener en cuenta es: a) la existencia o no de humedad o agua en circulación en contacto con la estructura; b) el contenido en oxígeno del terreno, y c) la existencia de corrientes erráticas que puedan dar lugar a fenómenos de corrosión por electrólisis.
La presencia en los suelos de sustancias que sean agresivas para el propio hormigón en masa, también producirá en último término la corrosión de las armaduras, ya que al agrietar y destruir el hormigón se producirá a continuación la oxidación de las mismas.

\section{CONCLUSION}

En los últimos años resulta alarmante el número creciente de estructuras de hormigón de corta edad con signos evidentes de un deterioro prematuro, hecho de enorme transcendencia socio-económica. Las peritaciones de las estructuras deterioradas han demostrado que tales hechos son siempre la consecuencia de una interacción no prevista entre la estructura y el medio ambiente, por lo que resulta esencial evaluar adecuadamente, ya a nivel de proyecto, su agresividad de cada ambiente.

La clasificación de la agresividad ambiental entraña dificultades; no obstante, en los últimos años se han realizado aproximaciones interesantes vinculando clasificaciones a procesos de deterioro concretos, que sería deseable que se incorporaran a la actual Normativa española cuando se proceda a su revisión.

\section{BIBLIOGRAFIA}

(1) CEB T. G. DURABILITY. "Durability of Concrete Structures. State of the Art. Report". CEB Bull, n. ${ }^{\circ} 148$, febrero, 1982.

(2) CEB T. G. n. ${ }^{\circ}$ 20. DURABILITY AND SERVICE LIFE OF C. S. "Draft-CEB-Guide to Durable Concrete Structures". CEB Bull. n. 166 , mayo, 1985.

(3) RASHEEDUZZAFAR nad al. "Deterioration of Concrete Structures in the Environment of the Middle east". ACl Journal, enero-febrero, 1984.

(4) FELIU, S. "Corrosión atmosférica de los materiales metálicos". Teoría y Práctica de la lucha contra la Corrosión. CSIC, Madrid, 1984.

(5) COSTA, J. M.; ESTAPE, A.; MIRO, E.; VILARRASA, M. "Contaminación Atmosférica por cloruros en Cataluña durante el periodo 1982/1985". II Jornades sobre Corrosió i Medi Ambient. Barcelona, 27-29 de mayo, 1986.

(6) FERNANDEZ, J. A.; PORRO, A. "Corrosión Atmosférica en el Pais Vasco. Inicio del Estudio". IV Congreso Español de Corrosión y Protección. Madrid, 11-14 febrero, 1986.

(7) ESPADA, L.; VILAR, J. C.; MERINO, P. "Determinación de la Corrosión Atmosférica del Acero al Carbono y Cobre en amplias Zonas Geográficas". IV Congreso Español de Corrosión y Protección. Madrid, 11-14 febrero, 1986.

(8) ESPADA, L.; SANCHEZ, A. M.; GONZALEZ, A.; NOVOA, $X$. R. "Corrosividad Atmosférica y Marina en las zonas Costeras Gallegas". IV Congreso Español de Corrosión y Protección. Madrid, 11-14 febrero, 1986. 
(9) COSTA, J. M.; PASTOR, E.; VILARRASA, M. "Corrosión de Aceros en Ambiente Marino". IV Congreso Español de Corrosión y Protección. Madrid, 11-14 febrero, 1986.

(10) VERBECK, G. J. "Mechanisms of Corrosion of Steel in Concrete". Corrosion of Metals in Concrete, ACI Publication SP-49, 3. ${ }^{a}$ edición. Detroit, Michigan, diciembre 1984.

(11) PEREPEREZ, B.; BARBERA, E.; GIMENEZ, E; GARCIA, L. "Intervenciones en Estructuras de Edificación de Hormigón Armado por Corrosión de las Armaduras". IV Congreso Español de Corrosión y Protección. Madrid, 11-14 febrero, 1986.

(12) ESPADA, L.; NOVOA, X. R.; VAZQUEZ, M. E. "Caracteristicas de la Corrosión del Aluminio en Zonas Urbanas, Rurales y Maritimas Gallegas". IV Congreso Español de Corrosión y Protección. 11-14 febrero, 1986.

(13) LAGO HELENE, P. R. DO. "Corrosión de las Armaduras en el Hormigón Armado". Cemento-Hormigón, n. ${ }^{\circ}$ 593, abril, 1983.
(14) G.J. QR.V. O. VENNESLAND Q . "Electrical resistivity of concrete in the oceans". 9th Annual off-shore Technology Conference. Houston, Texas. Mayo, 1977, pg. 581.

(15) TUUTTI, K. "Service Life of Structures with Regard to Corrosion of Embedded Steel". Quality Control of Concrete Structures, Vol. 1. Estocolmo, 17-21 junio, 1979.

(16) MOPU. "Instrucción para el proyecto y la Ejecución de Estructuras de Hormigón en Masa o Armado". Madrid, 1982.

(17) CEB/FIP. "CEB/FIP Model Code for Concrete Structures". 1978.

(18) METHA, K. "Durability of Concrete in Marine Environment-A Review". Performance of Concrete in Marine Environment, $\mathrm{ACl}$ Publication SP-65, 2. ${ }^{\mathrm{a}}$ Ed. Detroit, Michigan, abril, 1982.

(19) ACI COMMITTEE 201. "Guide for Making a Condition Survey of Concrete in Service. ACI 201, 1-68/79". ACI Journal, noviembre, 1968.

publicaciones del i.e.t.c.c.

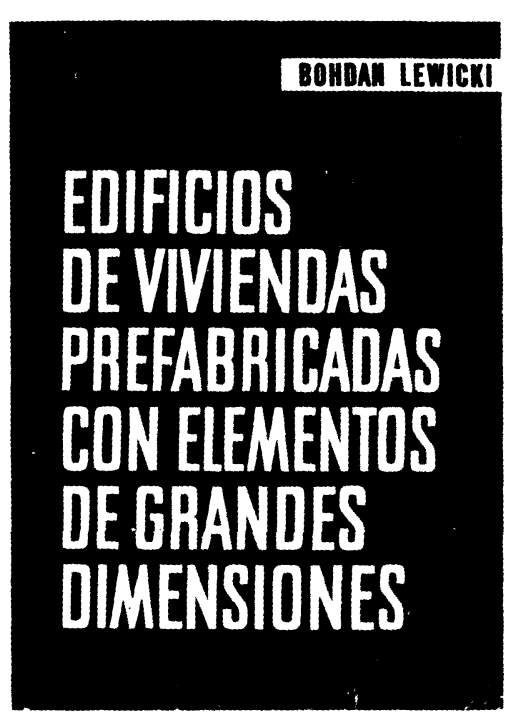

Bohdan Lewicki

Este libro trata de los problemas relativos a la construcción de los edificios de viviendas o publicos realizados con elementos prefabricados de grandes dimensiones. Se han estudiado los problemas de arriostramiento, asi como los que plantea la resistencia de los elementos y de la estructura; se han examinado las cuestiones de orden higrotermico, acústico y de resistencia al fuego; también se ha profundizado en el estudio de la estanquidad de los muros exteriores y de las juntas.

La obra incluye numerosas ilustraciones que dan detalles de diversas soluciones, asi como ejemplos de cálculo, tablas de valores numéricos, diagramas y ábacos.

Un volumen encuadernado en tela, de $24 \times 17 \mathrm{~cm}$, compuesto de 616 págs
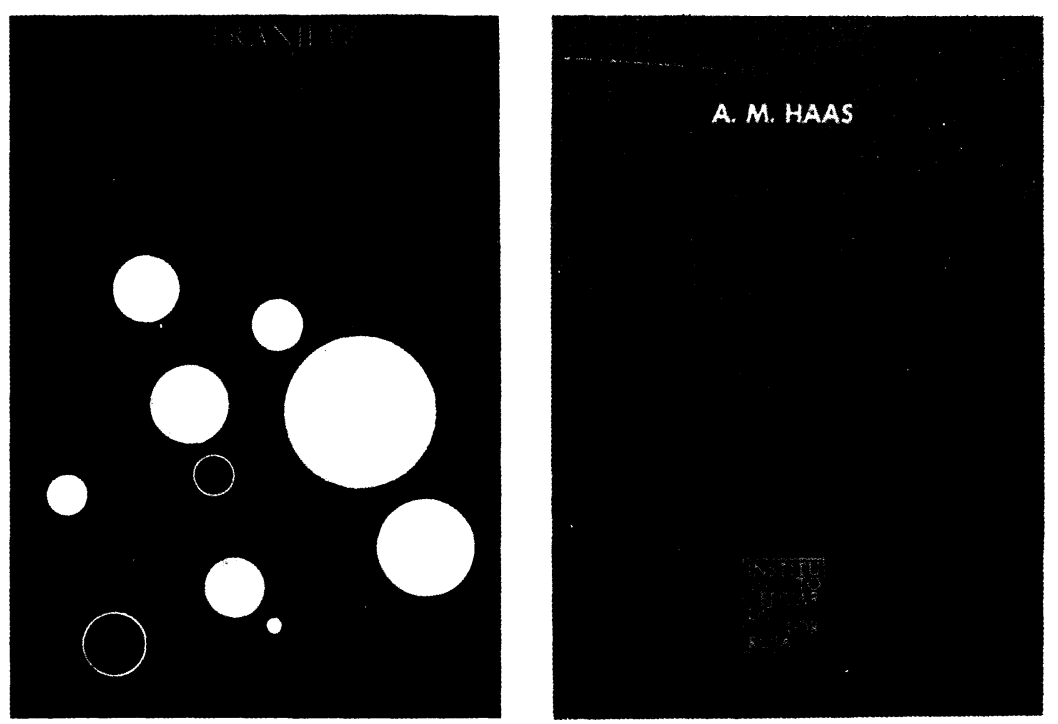

Zorislav Franjetic

En la obra de Franjetic se expone de una forma minuciosa, ordenada y sistemática todo un cuerpo de doctrina que reúne el conocimiento actual sobre el endurecimiento rápido del hormigón. Parte el autor de los principios básicos y llega a las últimas consecuencias y realidades técnicas y economicas.

Es una obra de consulta, tanto para el in vestigador sobre la materia, como para el proyectista y el realizador y montador de plantas e instalaciones y equipos de curado y endurecimiento rápido.

Un volumen encuadernado en cartóné, de $17 \times 24,5 \mathrm{~cm}$, compuesto de 385 págs. 110 figuras y 10 tablas.

Precios: 2.500 ptas.; SUSA 36.00.

\section{A. M. Haas}

Al escribir este libro el autor intento poner a disposición de los estudiantes y de los ingenieros unos conocimientos prácticos, adecuados para servir de guia en el diseño y construcción de láminas delgadas de hormigón.

El autor está convencido de que el éxito en el diseño de una lámina exige, por parte del proyectista, un examen de las tres fases por las que pasa la materialización de la lámina: el diseño, el análisis estructural y la cons trucción de la estructura.

Un volumen encuadernado en tela, de $17 \times 24,5 \mathrm{~cm}$, compuesto de 420 oágs., 141 figuras, 22 fotografias y 6 tablas.

Precios: 2.500 ptas.; SUSA 36.00 